\title{
Erratum to: Triangular ruthenium acetate clusters containing the bis(pyridyl)propane ligand and their inclusion chemistry with $\beta$-cyclodextrin
}

Sofia Nikolaou - Sérgio Hiroshi Toma •

Vagner Roberto de Souza $\cdot$ Juliano Alves Bonacin •

Koiti Araki · Henrique Eisi Toma

Published online: 21 October 2011

(C) Springer Science+Business Media B.V. 2011

Erratum to: Transition Met Chem (2011) 36:775-783

DOI 10.1007/s11243-011-9532-9

Unfortunately, the name of the fourth author of this paper was captured incorrectly. It is Juliano Alves Bonacin and not Juliano B. Alves.

The online version of the original article can be found under doi:10.1007/s11243-011-9532-9.

S. Nikolaou $(\bowtie)$

Departamento de Física e Química, Faculdade de Ciências Farmacêuticas de Ribeirão Preto da Universidade de São Paulo, Av. do Café s/número, Ribeirão Preto 14090-903, Brazil e-mail: sofian@fcfrp.usp.br

S. H. Toma · J. A. Bonacin · K. Araki · H. E. Toma ( $₫)$ Instituto de Química da Universidade de São Paulo-USP, P.O. BOX 26077, São Paulo 05513-970, Brazil

e-mail: henetoma@iq.usp.br

V. R. de Souza

Universidade Estadual de Maringá, Av. Colombo 5.790,

Maringá 87020-900, Brazil 